\title{
Vertebral body infection due to esophageal perforation caused by ingestion of fishbone: a case report
}

Ling-Yun Zhanga, Hong Liang ${ }^{\mathrm{b}}$, Xue-Guo Sun ${ }^{\mathrm{a}, *}$

aDepartment of Gastroenterology, The Affiliated Hospital of Qingdao University, Qingdao, Shandong 266000, China

${ }^{\circ}$ The Community Healthcare Center of Yan'an'er Road, Qingdao, Shandong 266000, China

Received: 12 June 2019; Accepted: 23 August 2019; Published: 20 June 2020

Abstract: Background: Esophageal injury is a common complication of foreign bodies in the upper gastrointestinal tract, but bilateral pleura effusion or vertebral infection is a rare condition due to a swallowed fishbone. It is considerably difficult for a physician to diagnose quickly because of incomplete patient history of foreign bodies ingestion and/or insufficient experiences.

Patient concerns: We describe the case of a 56-year-old man who was admitted to an emergency medical department owing to a low to moderate fever for 7 days. After a series of examinations, the patient was diagnosed with esophageal perforation (EP) caused by a fishbone that was swallowed half a month ago.

Diagnoses: About 12 days after the onset of fever, he was diagnosed with EP based on the gastric endoscopic images combined with histological section and sufficient history of the disease. About 2 months later, the patient has obvious back pain and lack of strength in two legs and was diagnosed with vertebral body infection.

Interventions: Antibiotic therapy, multi-disciplinary team (MDT), and surgical intervention had been exerted.

Outcomes: It is very fortunate for this patient to have a good prognosis due to a timely diagnosis and proper management. Muscle power has attained level 5.

Lessons: Several lessons can be learned from this case; for example, physicians should be alerted to the EP, endoscopic intervention should be prompt, antibiotics should be used regularly, and so on.

Keywords: esophageal perforation $\bullet$ spinal infection $\bullet$ foreign body $\bullet$ operation

(c) Shanxi Medical Periodical Press.

\section{Introduction}

Esophageal perforation (EP) was first described by Hermann Boerhaave who, in 1723, observed a spontaneous rupture of the esophagus occurring after repeated vomiting of an admiral in a Dutch Navy. ${ }^{1}$ It was not until 1947 that Barrett and Olson made the first attempts at surgical repair of EP. ${ }^{2,3}$ Despite update progress in intensive care, diagnostic methods, and endoscopic or surgical therapeutic measures of EP, the general mortality is still about $20 \% .{ }^{4}$ Diagnostic delays/errors due to frequent atypical clinical presentations/incomplete history, associated with the absence of clear management policies can partly explain this poor prognosis. Vertebral

How to cite this article: Zhang LY, Liang H, Sun XG. Vertebral body infection due to esophageal perforation caused by ingestion of fishbone: a case report. Front Nurs. 2020; 2: 77-80. 
infection is a rare clinical condition with relatively atypical manifestations and slow progress. The esophageal penetration of a swallowed fishbone is a much rarer case, though fishbone is a common foreign body in the gastrointestinal tract. We present a case of vertebral body infection caused by fishbone perforation, which has seldom been reported in the English literature.

\section{Case report}

On September 23, 2016, a 56-year-old man consulted the emergency department for 7 days with unknown fever. The highest temperature was $38.5^{\circ} \mathrm{C}$. Blood tests were as follows: WBC $7.75 \times 10 \%$ L, NEUT $76.94 \%$, CRP $120.57 \mathrm{mg} / \mathrm{L}$, and procalcitonin $0.325 \mathrm{ng} / \mathrm{ml}$. Posterior superior mediastinal masses with thickened adjacent esophagus wall and bilateral pleural effusion were revealed by thoracic computed tomography (CT) scan (Figure 1). A $1.0 \mathrm{~cm} \times 2.0 \mathrm{~cm}$ protuberant mass was found in the left wall of the esophagus at $20 \mathrm{~cm}$ from the incisors by the gastric endoscopy (Figure 2). Endoscopic biopsy shows a chronic active inflammation with

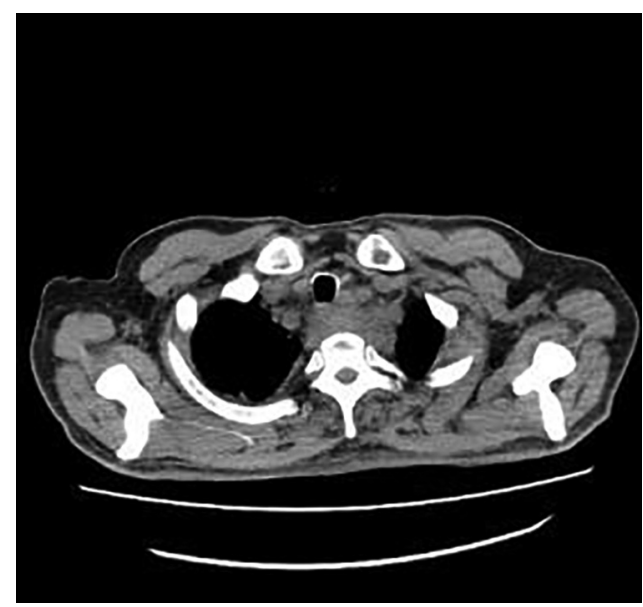

Figure 1. The CT scan result.

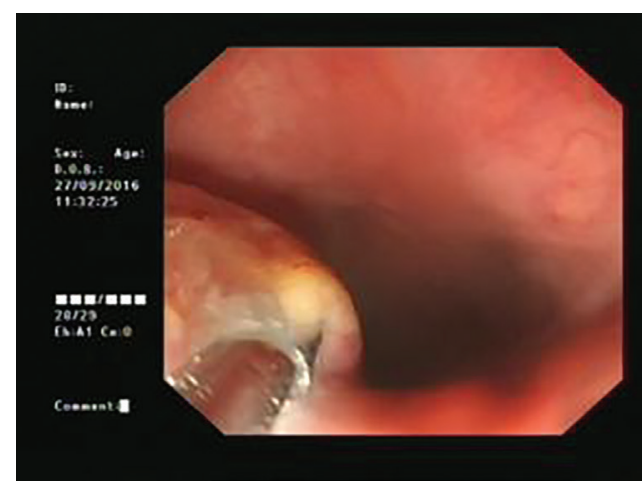

Figure 2. The gastric endoscopy result. ulcer and granulation tissue proliferation. Examine the medical history in detail at that moment, the patient was diagnosed with an odynophagia and admitted that he had accidentally swallowed a fishbone 2 months ago. Taken together, the patient was diagnosed with secondary mediastinitis due to esophagus perforation caused by the ingestion of fishbone. The patient had been treated moxifloxacin intravenously for 15 days, and then he was discharged without fever. Obvious back pain and decreased muscle strength began on the 7th day after discharge. On November 10, he had a Magnetic resonance (MR) of the thoracic vertebra, and the result was C7/T1 centrum infection and paravertebral abscess (Figure 3). The surgical intervention had been performed 4 days later. Consequently, the back pain disappeared, the muscle strength recovered, and an MR reexamination showed the patient recovered completely (Figure 4).

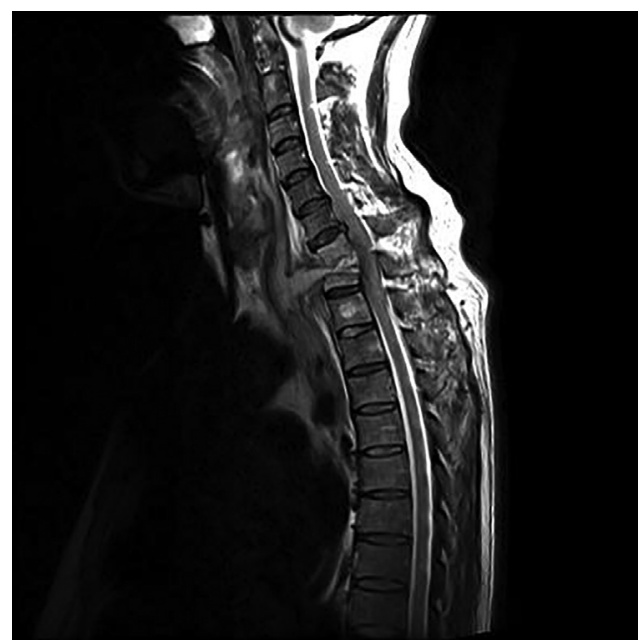

Figure 3. MR result of the thoracic vertebra before the operation.

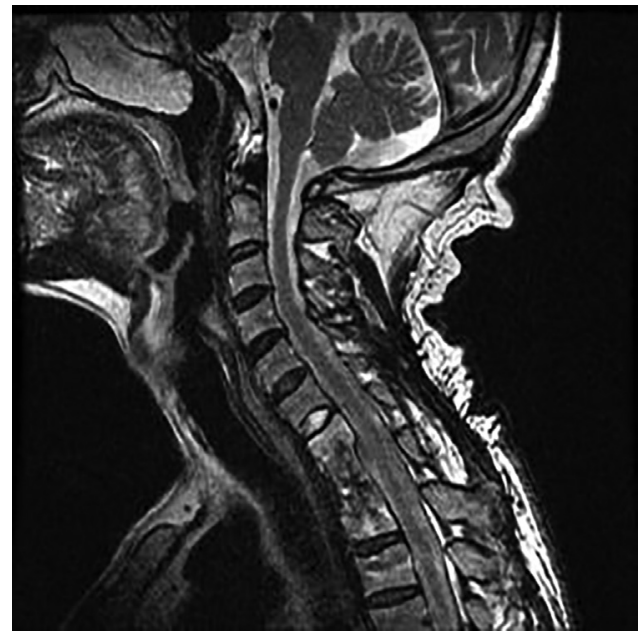

Figure 4. MR reexamination result of the thoracic vertebra after the operation. 


\section{Discussion}

Fishbone is perhaps the most commonly ingested foreign body located in the upper gastrointestinal tract and causes EP in humans. ${ }^{4}$ EP often led to a local infection of the esophagus, and mediastinitis would be developed with no proper treatment. Consequently, the infection might further spread to vertebral bodies as a rare clinical condition that many physicians cannot diagnose this condition timely. EP is a high-mortality condition, but early diagnosis and treatment can improve the prognosis. ${ }^{5}$ The essential attribute of the diagnostic approach to esophageal rupture is the maintenance of a high index of suspicion. ${ }^{6}$ Any patient who presents with fever or back pain weeks or even months after odynophagia should be aggressively evaluated,

\section{References}

1. Patel AR, Alton TB, Bransford RJ, et al. Spinal epidural abscesses: risk factors, medical versus surgical management, a retrospective review of 128 cases. Spine J. 2014;14:326-330.

2. Peters N, Upadhyay S, Grewal S, et al. Spinal epidural abscess following epidural cannulation for flail chest analgesia: a case report. Neurol Neurochir Pol. 2011;45:80-83.

3. Johnson KG. Spinal epidural abscess. Crit Care Nurs Clin N Am. 2013;25:389-397.

4. Shan GD, Chen ZP, Xu YS, et al. Gastric foreign body granuloma caused by an embedded to rule out perforation of the esophagus. The first lesson from this case is that close endoscopic and radiologic follow-up is mandatory to ensure early diagnosis and proper treatment for EP. Next, antibiotic treatment should be performed with adequate dosage and duration. Finally, the infected tissues from the esophagus should be cultured for bacteria, mycobacteria, and fungi to guide treatment.

\section{Ethical approval}

Ethical issues are not involved in this paper.

\section{Conflicts of interest}

All contributing authors declare no conflicts of interest.

fishbone: a case report. World J Gastroenterol. 2014;20:3388-3390.

5. Markar SR, Mackenzie H, Wiggins T, et al. Management and outcome of esophageal perforation: a national study of 2564 patients in England. Am J Gastroenterol. 2015;110:1559-1566.

6. Kang MS, Kim KH, Park JY, et al. Management of esophageal and pharyngeal perforation as complications of anterior cervical spine surgery. World Neurosurg. 2017;102:275-283. 